\title{
Vertical asymmetry of lamina cribrosa tilt angles using wide bandwidth, femtosecond mode-locked laser OCT; effect of myopia and glaucoma
}

\author{
Takuhei Shoji $^{1,2}$ (1) Hiroto Kuroda ${ }^{2}$ - Masayuki Suzuki ${ }^{2} \cdot$ Hisashi Ibuki ${ }^{1,2}$. \\ Makoto Araie $^{1,3} \cdot$ Shin Yoneya ${ }^{1,2}$
}

Received: 7 March 2016 /Revised: 21 September 2016 / Accepted: 11 October 2016 /Published online: 28 October 2016

(C) The Author(s) 2016. This article is published with open access at Springerlink.com

\begin{abstract}
Purpose Morphological features of the lamina cribrosa (LC) and optic disc may be important in the pathogenesis of glaucoma and myopic neuropathy. We therefore performed a cross-sectional study of patients with glaucoma and myopic neuropathy to evaluate vertical asymmetry of LC tilt angles (LCTAs) from Bruch's membrane opening (BMO).

Material and methods Forty-six control eyes and 35 primary open-angle glaucoma (POAG) eyes were included. A raster scanning protocol with 300 single B-scans (without averaging) were obtained using optical coherence tomography with a wide-bandwidth, femtosecond mode-locked (ML) laser. Superior temporal to inferior nasal (ST) direction and inferior temporal to superior nasal (IT) direction $\left( \pm 45^{\circ}\right.$ rotation with a horizontal line) lines were drawn, and the angle between the inner edge of the $\mathrm{BMO}$ plane and the best fitting line for the anterior LC plane was measured as the LCTA. The generalized estimating equation was used to analyze the eye-derived data. Results Although no significant differences in either STLTCAs or IT-LTCAs were observed between the glaucoma group and non-glaucoma group, the IT-LCTAs were found to be significantly greater than the ST-LCTA in both the glaucoma and non-glaucoma groups $(P<0.001)$. After adjustment for other potential confounding factors by multivariate
\end{abstract}

Takuhei Shoji

shoojii@saitama-med.ac.jp

1 Department of Ophthalmology, Saitama Medical University, 38 Morohongo Moroyama-machi, Iruma, Saitama 350-0495, Japan

2 Advanced Laser Medical Center, Department of Ophthalmology, Saitama Medical University, Iruma, Saitama, Japan

3 Department of Ophthalmology, Kanto Central Hospital, Tokyo, Japan analysis, greater refractive errors were significantly correlated with IT-LCTAs.

Conclusions Vertical asymmetry of the LC tilting from the BMO plane exists in both normal and POAG eyes, and correlates with the degree of myopia.

Keywords Mode-locked laser - Optical coherence tomography $\cdot$ Lamina cribrosa $\cdot$ Myopia $\cdot$ Glaucoma

\section{Introduction}

Myopia is an independent risk factor for glaucoma, and is frequently associated with atypical retinal nerve fiber layer (RNFL) defects and nerve vulnerability [1,2]. Furthermore, distortion of the lamina cribrosa (LC) is considered to be a major factor contributing to nerve vulnerability in myopia. Myopic eyes, particularly high myopic eyes, have stretching stress at the LC and at the edge of disc and sclera, which can cause susceptibility to nerve fiber damage [3, 4]. LC has also long been considered the primary site of axonal injury in glaucoma. Deformation and displacement of the LC has been increasingly implicated as the primary pathophysiological mechanism underlying glaucomatous optic neuropathy [5].

Optical coherence tomography (OCT) is a non-invasive optical imaging modality that allows real-time structural imaging of the fundus. Spectral-domain (SD) detection technology, combined with improvements in light sources incorporating a wider range of wavelengths (approximately $50 \mathrm{~nm}$ ), has facilitated the development of currently available SDOCT devices with three-dimensional (3D) imaging capability and high resolution $(5-7 \mu \mathrm{m})$. Advancements in OCT have enabled detailed examinations of the deep optic nerve head $(\mathrm{ONH})$, including the LC. In the clinical evaluations of $\mathrm{ONH}$, the termination of Bruch's membrane is considered the most 
consistent site from which to quantify the neuroretinal rim, and Bruch's membrane opening (BMO) as detected by SDOCT is considered a stable landmark and the most consistent outer border of the neuroretinal rim [6,7]. Although posterior displacement between BMO and the anterior surface of the LC has been well studied in glaucoma [8,9], there have been relatively few in-vivo studies demonstrating other anatomical parameters associated with $\mathrm{BMO}$ and $\mathrm{LC}$ in human eyes.

We identified that Bruch's membrane terminates with single B-scanning (without averaging) using a high-resolution OCT system and a light source spectral bandwidth of $200 \mathrm{~nm}$ with relative ease [10]. High-resolution image acquisition enabled faster clearance of single-scanned images, resulting in substantially faster 3D image construction than that obtained with the standard commercial SD-OCT instruments that are currently available [11]. Using this system, we previously reported a novel indicator of LC tilt angles (LCTAs), which correlated with both glaucoma and refractive errors [12].

The purpose of the present study was two-fold, i.e., to investigate the vertical asymmetry of LCTAs, and to evaluate any potential correlations with disorders such as glaucoma and with refractive errors.

\section{Material and methods}

The Ethics Committee of Saitama Medical University approved this cross-sectional comparative study, which was conducted in accordance with the tenets of the Declaration of Helsinki. Patients were included if they were at least 20 years old, fulfilled the eligibility requirements detailed below, and signed an informed consent form at a visit between April 2012 and July 2012.

\section{Inclusion criteria}

To be eligible for this study, patients with glaucoma had to have been previously diagnosed with POAG involving characteristic glaucomatous $\mathrm{ONH}$ damage, such as localized or diffuse neuroretinal rim thinning associated with glaucomatous loss of the visual field, in accordance with the criteria of Anderson and Patella [13] using a Humphrey Field Analyzer (Carl Zeiss Meditec, Dublin, CA, USA) and the standard 30-2 program of the Swedish Interactive Threshold algorithm.

Control subjects were enrolled during the study period; they had to have intraocular pressures between 10 and $21 \mathrm{mmHg}$, no family history of glaucoma, a normal open anterior chamber angle, clinically normal appearance of the optic disc, and normal visual field results [13].

The subjects were divided into a highly myopic group (spherical equivalent, equal or worse than $-5.0 \mathrm{D}$ ) and a non-highly myopic group (spherical equivalent, better than $-5.0 \mathrm{D}$ ) on the basis of spherical equivalents.

\section{Exclusion criteria}

Exclusion criteria were as follows: (1) visual acuity worse than $20 / 40$, (2) poor reliability on visual field test result ( $>20 \%$ fixation loss or $>15 \%$ false positive or false negative answers), (3) any other ophthalmic disease, including media opacity, diabetic retinopathy, neuro-ophthalmological disease, uveitis, ocular trauma, retinal, or choroidal disease, (4) other diseases capable of causing VF loss or optic nerve deterioration or a history of intraocular surgery or laser treatment, or (5) eyes with an extremely tilted or anomalous disc.

\section{Instruments}

The OCT system was built by the Advanced Laser Medical Center (ALMC) at Saitama Medical University. The details of our SD-OCT system have been previously described elsewhere $[11,12,14]$. In brief, the OCT system used an ultrabroadband, Kerr lens, mode-locked (ML) Ti:sapphire laser, and a wide-band spectrometer. The spectral bandwidth of the light source was $200 \mathrm{~nm}$ full width at a central wavelength of $840 \mathrm{~nm}$. A high speed CCD camera with $2048 \times 300$ pixels (Basler, Ahrensburg, Germany) was used as the detection system. The measurement speed was 50,000 depth-scans/s, and depth resolution was measured to be less than $2.0 \mu \mathrm{m}$ into the tissue $[12,14]$. The interferometer was attached to a semicustom fundus scanning head system.

\section{Acquisition of in-vivo three-dimensional OCT images}

A raster scanning protocol with 300 single B-scans with 300 A-scans (with 2048 pixels/A-Scan) covering a $3.0 \times 3.0 \mathrm{~mm}$ square region centered on $\mathrm{ONH}$ was used for volumetric scans. Volumetric rendering of the 3D-OCT data set was performed, and longitudinal and en face cross-sections were constructed using an image processing software (Amira 5.4.3, Mercury Computer Systems Inc., Chelmsford, MA, USA). A fundus image was generated as an en face projection image from the 3D data set by integrating the magnitudes of the OCT signals at each lateral position along the axial direction. The total data acquisition time for a single 3D-OCT volumetric image was $3.0 \mathrm{~s}$. OCT images were acquired after pupil dilation with tropicamide and phenylephrine hydrochloride (Mydrin P; Santen, Osaka, Japan). The optic disc was also imaged using a digital $30^{\circ}$ fundus camera (Zeiss FF450, Carl Zeiss, Jena, Germany) immediately prior to OCT data acquisition. 


\section{LCTA measurement}

The measurement of LCTAs was conducted as previously reported [12]. Briefly, following acquisition of an in-vivo 3D dataset, lines were drawn in a superior temporal (ST) to inferior nasal (IN) direction and inferior temporal (IT) to superior nasal (SN) direction $\left( \pm 45^{\circ}\right.$ rotation with the horizontal line). B-scan images of the ST-IN and the IT-SN directions were reviewed. Figure 1 shows a schematic explanation of the methodology used for measuring LCTA against BMO. We defined the ST-IN LCTAs (ST-LCTA) and IT-SN LCTAs (IT-LCTA) as follows. The center of the B-scan (white dotted line) was defined as the intermediate scan between the interruption where the Bruch's membrane line started. A line (red dotted line) connecting the proximal tips of Bruch's membrane on each side of the ON head on a cross sectional SDOCT image was drawn and set as the BMO reference plane. LCTA was defined as the angle between the inner edge of the $\mathrm{BMO}$ plane, and the best-fitting line for the anterior LC plane measured as LCTA. The anterior surface of LC on B-scans was defined as the highly reflective plate structure underlying the optic disc cup. The definition of anterior surface of LC has been used in a previous ophthalmological study [15]. Angle measurements were performed with Amira software.

Measurements were repeated three times and averaged to provide final LCTA values. The intraobserver and interobserver reproducibility of LCTA measurements were evaluated in ten randomly selected eyes from five glaucomatous eyes and five normal eyes. Analysis was based on three independent series of re-evaluations made by two independent observers. The agreement of a single observer's measurement and the mean of all three measurements conducted by the two observers were calculated with the intraclass correlation coefficient (ICC) using a 2-way mixed effect model.

\section{Statistical analyses}

Data are expressed as means and the standard deviation (SD), with medians and interquartile ranges for continuous variables, and as frequencies with percentages for categorical variables. Baseline characteristics were summarized according to treatment sequences and compared using an unpaired $t$-test, the Wilcoxon rank sum test for continuous variables, or the chi-square test or Fisher's Exact test for categorical variables, as appropriate. As a number of subjects had both eyes included in the study, the generalized estimating equation was used to analyze data derived from these eyes. This model was also used to evaluate the mean difference between the groups and has been used in a previous ophthalmological study [16].

To determine the effects of various factors on IT-LCTA and ST-LCTA, we performed univariate and multivariate regression analyses. The model was adjusted for candidate factors including age, sex, glaucoma, and refractive errors. Coefficients with $95 \%$ confidence intervals (95\% CIs) were calculated. A $P$-value less than 0.05 indicated a statistically significant difference. All statistical analyses were performed using JMP version 10.1 software (SAS Institute, Inc., Cary,

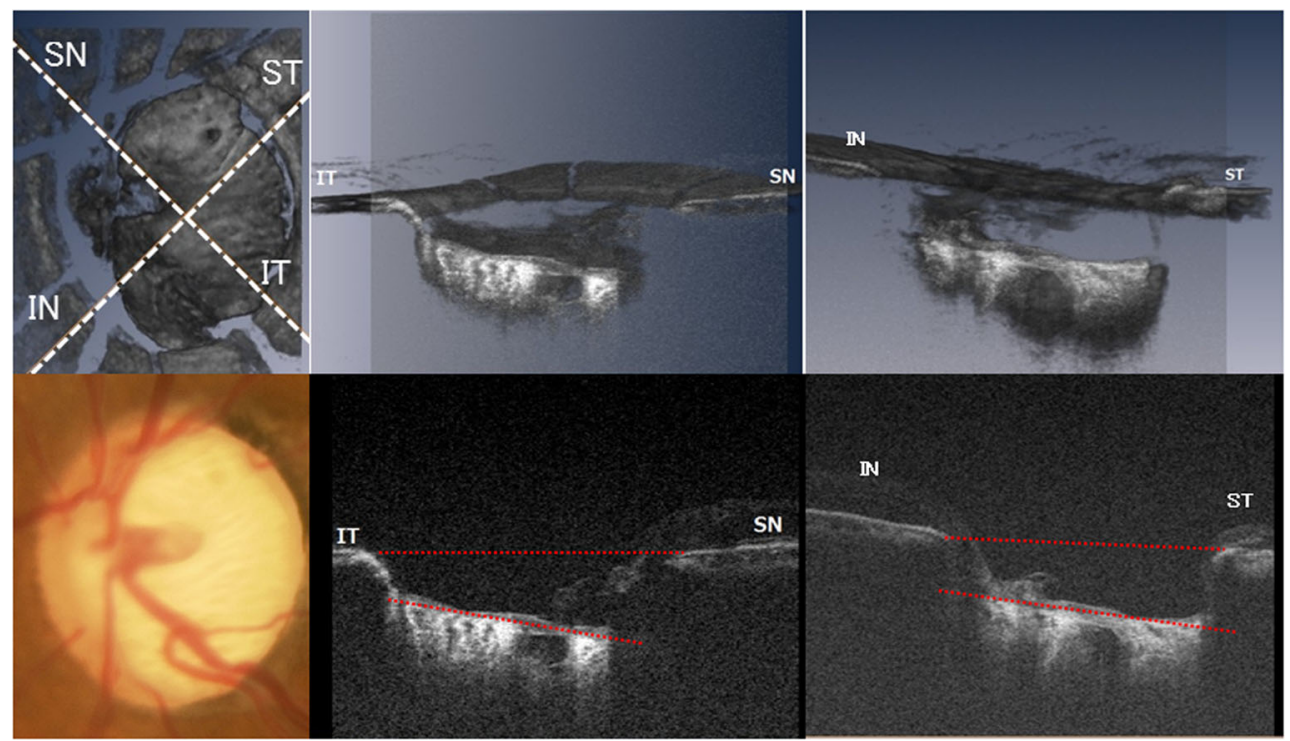

Fig. 1 A schematic explanation of the methodology used for measuring the lamina cribrosa tilt angles (LCTA) against the Bruch's membrane opening $(B M O)$. Top left: lines were drawn in the superior temporal $(S T)$ to inferior nasal $(I N)$ and inferior temporal (IT) to superior nasal $(S N)$ directions $\left( \pm 45^{\circ}\right.$ rotation with the horizontal line). Bottom left, white dotted line: fundus photography (top center and right) volume image sectioned at the level of IT to SN direction and ST to IN direction reconstructed from the 3D OCT dataset (bottom center and right) B-scans along IT to SN and ST to IN directions. A line (red dotted line) connecting the proximal tips of Bruch's membrane on each side of the ON head on the cross-sectional SD-OCT image was drawn and set as the BMO reference plane. LCTA was defined as the angle measured between the inner edge of the BMO plane and the best-fitting line (red dotted line) for the anterior LC plane 
NC, USA) and SPSS software, version 22 (Japan IBM, Tokyo, Japan).

\section{Results}

A total of 84 eyes from 48 participants were enrolled in the study. Of these eyes, three were excluded due to improper OCT images owing to media opacities and inappropriate fixation. Therefore, 81 eyes (from 46 participants), 46 normal eyes (25 subjects) and 35 primary open-angle glaucoma (POAG) eyes (21 patients), were included in the study. Table 1 summarizes the baseline characteristics of the study subjects. Mean LCTA measurements demonstrated excellent intra-observer $(\mathrm{ICC}=0.930$ for observer 1 and $\mathrm{ICC}=0.969$ for observer 2) and inter-observer (ICC $=0.983$ ) agreement (all $P<0.001$ ). Table 2 shows LCTAs from the BMO plane in both groups. The median (interquartile range) ST-LCTA and IT-LCTA were 3.2 (1.6-5.7) degrees and 10.1 (7.212.2) degrees respectively, in no glaucoma eyes, and 3.9 (2.6-6.4) degrees and 11.0 (5.5-18.8) degrees respectively, in glaucomatous eyes. Although no significant differences in either ST-LCTA or IT-LCTA were observed between the glaucoma group and non-glaucoma group, the IT-LCTAs were found to be significantly greater than the ST-LCTAs in both the glaucoma and non-glaucoma groups $(P<0.001)$. Moreover, although the difference in ST-LCTAs between group with high myopia and group without high myopia was not significant in both the glaucoma group and nonglaucoma group, IT-LCTAs in the group with high myopia were significantly greater than the group without high myopia in both the glaucoma group and non-glaucoma group. Table 3 shows the correlation between LCTAs and clinical and biometric parameters. Although there was no correlation between age, IOP, MD, or any LCTAs, there was a significant association between greater refractive errors with IT-LCTA and differences between ST-LCTA and IT-LCTA. Figure 2 shows representative cases. The top images are the hypertrophic eyes from a 61-year-old male patient without glaucoma. The middle images are from highly myopic eyes with glaucoma. The refractive errors were $-6.00 \mathrm{D}$. The bottom images are from the moderately myopic eyes with severe glaucoma. The visual field mean deviation was -15.52 decibels. IT-LCTA was greater than ST-LCTA in these three images. Figure $3 \mathrm{a}$ and $\mathrm{b}$ show the scatter plots of refractive errors and IT-LCTA and ST-LCTA values. The IT-LCTA values were positively correlated with refractive errors in both the non-glaucoma group $\left(R^{2}=0.127, P=0.015\right)$ and the glaucoma group $\left(R^{2}=0.346\right.$, $P<0.001$ ), but no significant correlation was observed between the ST-LCTA and refractive errors. Figure $3 \mathrm{c}$ shows the scatter plots for ST-LCTA and IT-LCTA values. Figure $3 \mathrm{~d}$ shows the scatter plot of significantly correlated differences between ST-LCTA and IT-LCTA values and refractive errors $(P<0.001)$. Table 4 describes the results of the multivariate analyses for factors potentially affecting STLCTA and IT-LCTA values. After adjusting for other potential confounding factors by multivariate analysis, greater refractive errors were significantly correlated with IT-LCTA (coefficient, -1.27 per diopter; $P<0.001)$ and the differences between IT-LCTA and ST-LCTA (coefficient, -1.14 per diopter; $P<0.001)$. The interactions between refractive errors and POAG did not show statistically significant correlations with IT-LCTA using multivariate analyses.

\section{Discussion}

The principal findings of this report were that the inferotemporal LCTA (IT-LCTA) was significantly larger than the superotemporal LCTA (ST-LCTA), and greater IT-LCTA and differences between them were significantly correlated with greater refractive errors after adjustment for other potential confounding factors, including presence of POAG. These results indicate that morphological features of LC, namely vertical asymmetry LC tilting in the IT-SN direction with reference to $\mathrm{BMO}$, were correlated with refractive errors. In contrast, no correlation was found between LC tilting in the ST-IN direction, and either presence of POAG or refractive errors.

The measurements in the present study were facilitated by SD-OCT with a wide-broadband light source that was able to depict the surface of the $\mathrm{LC}$ and BMO with a single scan with less than $2 \mu \mathrm{m}$ axial resolution. In a report by Park et al. [17],
Table 1 Baseline characteristics of the group without glaucoma and the group with glaucoma

\begin{tabular}{llll}
\hline & No glaucoma group $(n=46)$ & Glaucoma group $(n=35)$ & $P$ value \\
\hline Male sex $(n, \%)$ & $33(71.7)$ & $23(63.9)$ & 0.631 \\
Age (years) & $44.5 \pm 16.9$ & $63.1 \pm 8.4$ & $<0.001$ \\
Spherical equivalent error (D) & $-2.2 \pm 2.3$ & $-3.7 \pm 4.1$ & 0.067 \\
IOP (mmHg) & $14.9 \pm 2.3$ & $17.2 \pm 5.2$ & 0.010 \\
MD (dB) (median [25,75 percentile]) & $0.08(-1.76,0.73)$ & $-11.2(-5.0,-18.12)$ & $<0.001$ \\
\hline
\end{tabular}

Plus-minus values are means $\pm \mathrm{SD}$

Baseline characteristics were compared with the unpaired t-test between the groups

Abbreviations: $D$ diopters, $I O P$ intraocular pressure, $M D$ mean deviation, $d B$ decibels 
Table 2 Lamina cribrosa tilt angles in each group

\begin{tabular}{|c|c|c|c|c|c|}
\hline & \multicolumn{5}{|c|}{ LC tilt angle from BMO plane (deg.) (median $[25,75$ percentile $]$ ) } \\
\hline & $\begin{array}{l}\text { Superotemporal to } \\
\text { inferonasal direction }\end{array}$ & $\begin{array}{l}P \\
\text { value* }\end{array}$ & $\begin{array}{l}\text { Inferotemporal to } \\
\text { superonasal direction }\end{array}$ & $\begin{array}{l}P \\
\text { value* }\end{array}$ & $\begin{array}{l}P \\
\text { value }^{\dagger}\end{array}$ \\
\hline \multicolumn{6}{|l|}{ No glaucoma group } \\
\hline Overall $(n=46)$ & $3.2(1.6,5.7)$ & & $10.1(7.2,12.2)$ & & $<0.001$ \\
\hline $\begin{array}{l}\text { No high myopia } \\
\quad(n=39)\end{array}$ & $3.3(1.6,5.7)$ & & $9.2(7.2,11.7)$ & & $<0.001$ \\
\hline $\begin{array}{l}\text { High myopia } \\
\quad(n=7)\end{array}$ & $2.8(1.4,7.6)$ & & $12.1(7.9,16.3)$ & & 0.016 \\
\hline$P$ value & 0.965 & & 0.030 & & \\
\hline \multicolumn{6}{|l|}{ Glaucoma group } \\
\hline Overall $(n=35)$ & $3.9(2.6,6.4)$ & 0.176 & $11.0(5.5,18.8)$ & 0.300 & $<0.001$ \\
\hline $\begin{array}{l}\text { No high myopia } \\
\quad(n=22)\end{array}$ & $3.2(2.4,6.4)$ & 0.428 & $7.9(4.0,16.4)$ & 0.625 & $<0.001$ \\
\hline $\begin{array}{l}\text { High myopia } \\
\quad(n=13)\end{array}$ & $5.2(2.7,7.4)$ & 0.428 & $15.6(10.9,22.8)$ & 0.322 & $<0.001$ \\
\hline$P$ value & 0.657 & & 0.012 & & \\
\hline
\end{tabular}

Abbreviations: $L C$ lamina cribrosa, $B M O$ Bruch's membrane opening, deg degrees

* $P$ value for no glaucoma group vs glaucoma group

$\dagger P$ value for superotemporal to inferonasal direction vs inferotemporal to superonasal direction

$\ddagger P$ value for no high myopia group vs. high myopia group it took 10-20 min to construct a 3D image, which may preclude the use of commercial SD-OCT instruments for screening purposes. The theoretical advantage of a ML laser compared with superluminescent diodes (SLD) as an OCT light source [14] made the total data acquisition time for a single 3D-OCT (volumetric) image with shorter than the SD-OCT instrument using SLD. Although commercial swept-source (SS)-OCT instruments with a central wavelength of $1050 \mathrm{~nm}$ are able to provide deep penetration to the LC, the axial resolution is inferior to commercial SD-OCT instruments using SLD (see manufacturer's homepage at http://www.topcon-medical.eu/eu/products/177-dri-oct-1atlantis-swept-source-oct.html\#specifications) due to the bandwidth and central wavelength of the light source.

Myopia has been reported to be associated with optic disc tilting [18]. Kim et al. suggested tilted optic discs in myopic eyes may be an acquired feature resulting from scleral stretching in the parapapillary region associated with myopic shift [19]. In some myopic eyes, particularly highly myopic eyes, a number of abnormalities may be observed around the $\mathrm{ONH}$, including peripapillary conus, intrachoroidal cavity, optic disc pit, and focal LC defects [20-22]. Although the pathogenesis of peripapillary intrachoroidal cavity has yet to be fully elucidated, the intrachoroidal cavity commonly involves the area inferior to the optic disc [23]. Our observations also have implications for the current understanding of the pathogenesis of peripapillary intrachoroidal cavity in highly myopic eyes, as intrachoroidal cavity has previously been considered a temporal $\mathrm{ONH}$ change due to axial elongation.

Focal LC defects were defined as defects of continuous LC hyper-reflectivity. Although the locations of these focal defects corresponded with those of RNFL defects and disc
Table 3 Association of potential clinical and biometric parameters with the lamina cribrosa tilt angle

\begin{tabular}{|c|c|c|c|c|c|c|}
\hline \multirow[b]{3}{*}{ Variables } & \multicolumn{6}{|c|}{ Lamina cribrosa tilt angle } \\
\hline & \multicolumn{2}{|c|}{$\begin{array}{l}\text { Superotemporal to } \\
\text { inferonasal direction }\end{array}$} & \multicolumn{2}{|c|}{$\begin{array}{l}\text { Inferotemporal to } \\
\text { superonasal direction }\end{array}$} & \multicolumn{2}{|c|}{$\begin{array}{l}\text { Difference between IT- } \\
\text { LCTA and ST-LCTA }\end{array}$} \\
\hline & Coefficients & $P$ value & Coefficients & $P$ value & Coefficients & $P$ value \\
\hline Age (years) & 0.02 & 0.419 & -0.01 & 0.771 & -0.03 & 0.496 \\
\hline IOP (mmHg) & -0.05 & 0.571 & -0.05 & 0.809 & 0.00 & 0.983 \\
\hline $\mathrm{MD}(\mathrm{dB})$ & -0.12 & 0.073 & -0.08 & 0.558 & 0.04 & 0.779 \\
\hline Refractive errors (SEQ) & -0.07 & 0.529 & -1.12 & $<0.001$ & -1.05 & $<0.01$ \\
\hline
\end{tabular}

Abbreviations: $I O P$ intraocular pressure, $M D$ mean deviation, $d B$ decibels, $S E Q$ spherical equivalent, $D$ diopter, $I T$ inferotemporal to superonasal direction, $S T$ superotemporal to inferonasal direction 


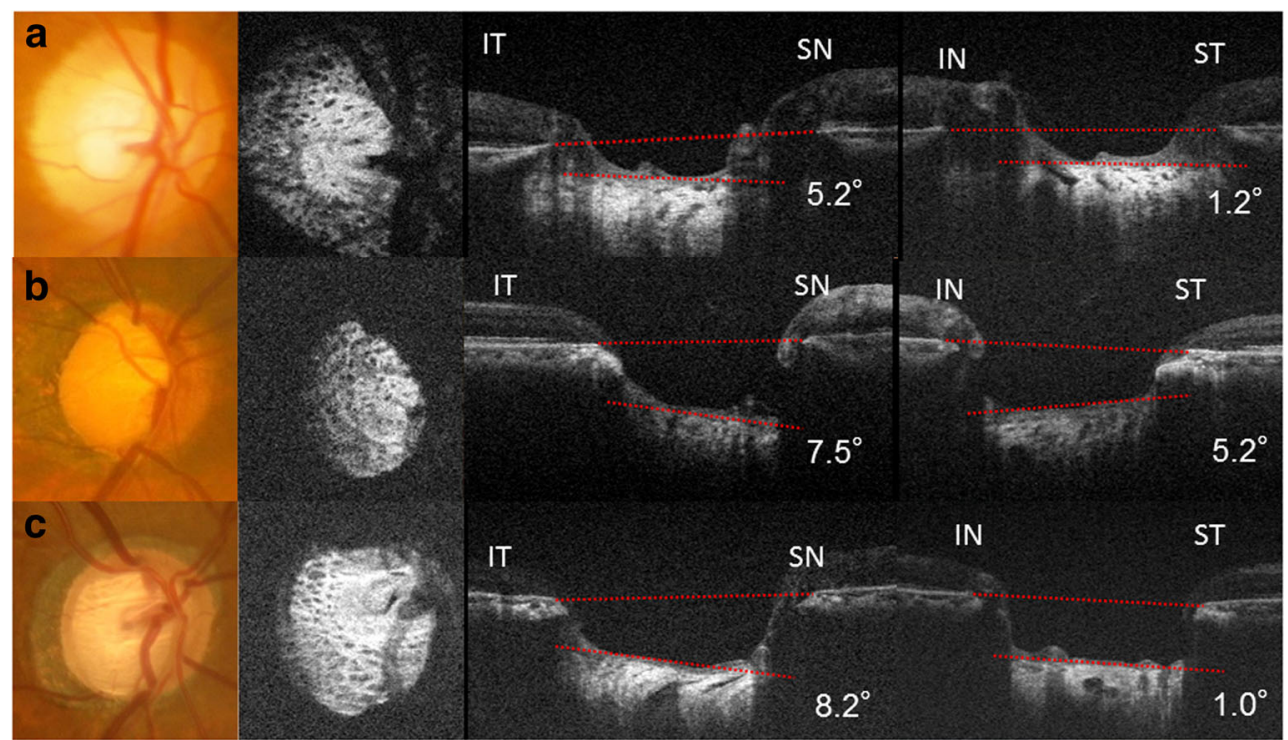

Fig. 2 Fundus images and en face images from optical coherence tomography $(O C T)$, and inferotemporal $(I T)$ to superonasal $(S N)$ direction and inferonasal $(I N)$ to superotemporal $(S T)$ direction B-scan images from optical coherence tomography $(O C T)$. a (top) Images from the right eye of a 61-year-old male without glaucoma. The refractive error was $+1.25 \mathrm{D}$ (top right). The LC tilting was mild in both the IT-SN direction (IT-LCTA) and the ST-IN direction (ST-LCTA). b (middle left) Images from the right eye of a 71-year-old male with glaucoma.

hemorrhages [21], the mechanism of focal LC defect formation has yet to be elucidated. However, the majority of focal LC defects occurred in the inferior or inferotemporal periphery of LC [24], and the frequency of LC defects was much higher in eyes with high myopia than in those without. Recently, Kimura et al. reported that the formation of LC defects may be associated with temporal elongation of the optic disc; he demonstrated that the presence of LC defects was significantly associated with disc deformation, such as vertical "disc" tilt angle, by multivariate analysis [25]. Our results corroborate these previous findings, demonstrating that structural changes in the inferotemporal region are associated with myopia. In this study, greater refractive errors were correlated with greater IT-LCTA, possibly due to stretching or deformation, involving lamina tilt in the axial direction in addition to disc tilt. We previously reported that LCTA were correlated with glaucoma in the vertical direction and refractive errors in the horizontal direction [12]. We hypothesize that LC tilting from the BMO plane, particularly in the inferotemporal direction due to refractive errors, might be associated with deformation around the disc and LC such as peripapillary ICC or focal LC defects. Further, the inferotemporal sector of the neuroretinal rim is the most significant sector in patients with glaucoma. Nevertheless, the detailed mechanisms underlying these abnormalities remain unclear, with these theories currently remaining hypothetical. Further studies are required to fully evaluate any correlation between lamina tilt and disc deformation.
The refractive errors were $-6.00 \mathrm{D}$ and the visual field mean deviation was -15.22 decibels. Both IT-LCTA (middle center) and ST-LCTA (middle right) were evident. c (bottom left) Images from the right eye of a 73-year-old male with glaucoma. The refractive errors were $-1.00 \mathrm{D}$, and the visual field mean deviation was -15.52 decibels. IT-LCTA was greater than ST-LCTA. IT-SN (bottom center) and ST-IN (bottom right) views of the B-scan image

We used BMO as a reference plane to measure LCTA, but not the photographed disc margin, because photographically determined clinical disc margins reportedly represent complex anatomical structures that differ between individual eyes [6, 26, 27]. On the other hand, BMO detected by SD-OCT is considered to be the most consistent outer border of the neuroretinal rim and a stable landmark $[6,7]$. Three-dimensional imaging allowed measurement of LCTA from the BMO plane along the ST to inferonasal and along the inferotemporal to superonasal lines through the optic disc center, determined by using the edges of $\mathrm{BMO}$ on the $3 \mathrm{D}$ dataset as references. However, BMO, in addition to scleral openings, might also be tilted in myopic eyes. It remains to be determined whether BMO tilting and LC tilting in myopia are dependent or independent phenomena. Our findings, taken together with previous findings, suggest that myopia results in LC deformation against BMO in addition to altering disc configuration on $\mathrm{ONH}$.

Several limitations of this study warrant discussion. First, this was a pilot study involving a small number of subjects and had a cross-sectional design. The effect of progression of glaucomatous neuropathy on the LCTA might be underestimated because of the small number of cases and undetected progression of glaucoma. Refractive errors and age were not matched between the no-glaucoma group and the glaucoma group. To control the effects of age differences, we adjusted for potential 
Fig. 3 Scatterplot graphs of a ITLCTA versus refractive errors and b ST-LCTA versus refractive errors. c Scatterplot graphs of ITLCTA versus ST-LCTA. d Scatterplot graphs of the difference between ST-LCTA and IT-LCTA versus refractive error. Circle, with glaucoma; cross, without glaucoma a

a IT-LCTA (inferotemporal to superonasal )
and refractive errors (deg.)

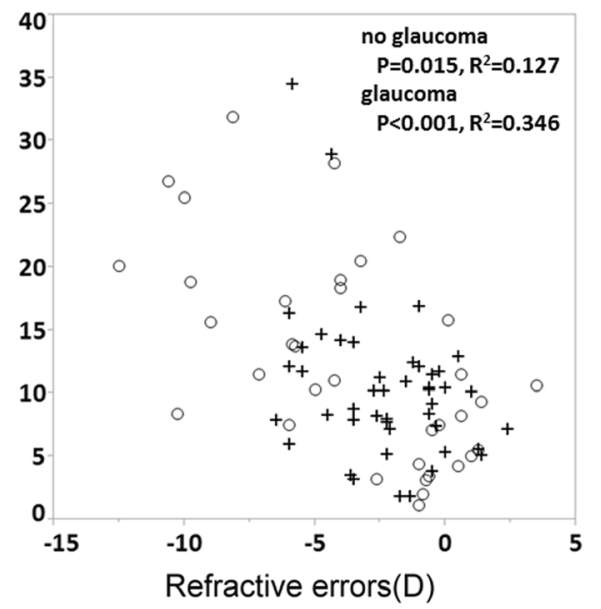

c

ST-LCTA and IT-LCTA

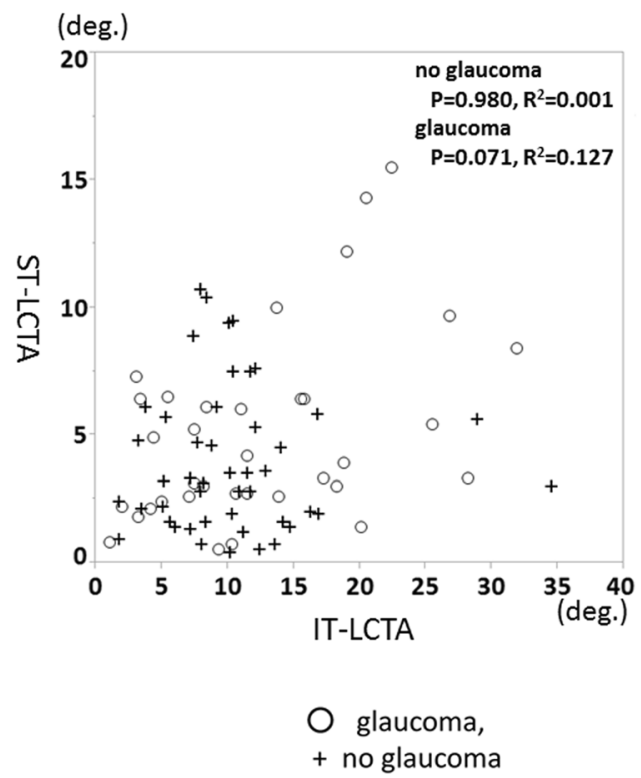

b ST-LCTA (superotemporal to inferonasal) (deg) and refractive errors

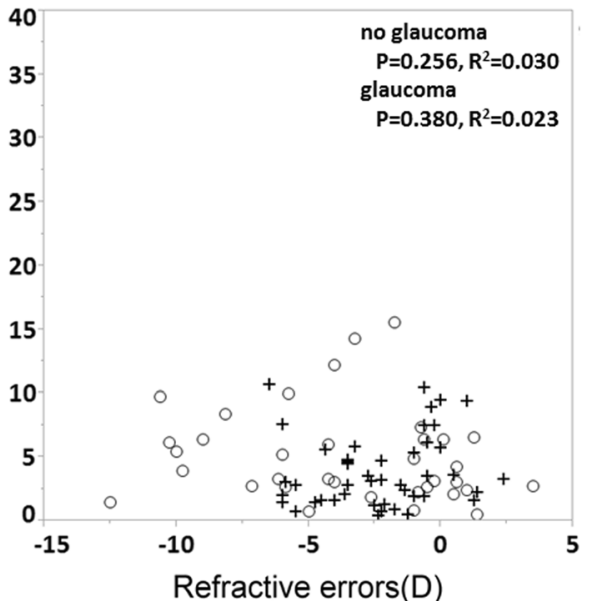

d $\Delta(\mathrm{ST}-\mathrm{LCTA})-(\mathrm{IT}-\mathrm{LCTA})$ and refractive errors

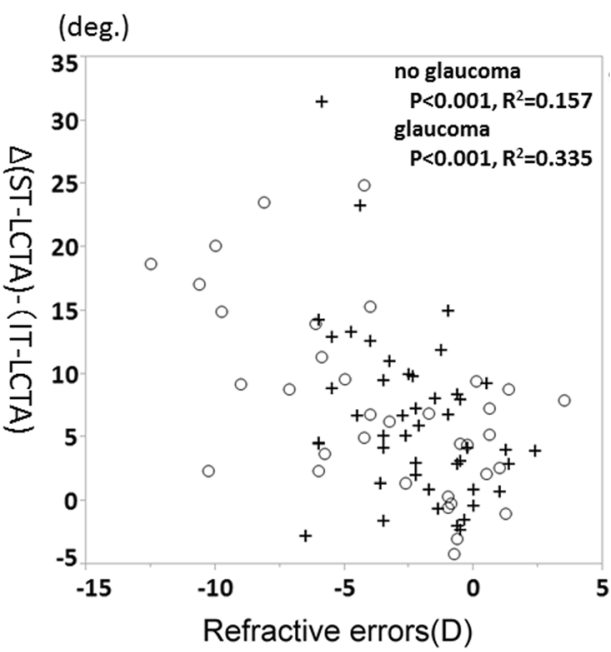

Table 4 Association of potential clinical and biometric parameters with the lamina cribrosa tilt angle based on multivariate analyses

\begin{tabular}{|c|c|c|c|c|c|c|}
\hline \multirow[b]{2}{*}{ Variables } & \multicolumn{2}{|c|}{$\begin{array}{l}\text { Superotemporal to inferonasal } \\
\text { direction }\end{array}$} & \multicolumn{2}{|c|}{$\begin{array}{l}\text { Inferotemporal to superonasal } \\
\text { direction }\end{array}$} & \multicolumn{2}{|c|}{$\begin{array}{l}\text { Difference between IT-LCTA and ST- } \\
\text { LCTA }\end{array}$} \\
\hline & $\begin{array}{l}\text { Coefficients } \\
(95 \% \text { CI })\end{array}$ & $P$ value & $\begin{array}{l}\text { Coefficients } \\
(95 \% \text { CI })\end{array}$ & $P$ value & $\begin{array}{l}\text { Coefficients } \\
(95 \% \text { CI })\end{array}$ & $P$ value \\
\hline Age (per year) & $0.02(-0.09,0.12)$ & 0.755 & $0.11(-0.16,0.38)$ & 0.422 & $0.10(-0.16,0.35)$ & 0.459 \\
\hline Sex (reference: male) & $-1.62(-3.96,0.71)$ & 0.173 & $0.31(-5.63,6.24)$ & 0.919 & $1.93(-3.52,7.38)$ & 0.487 \\
\hline $\mathrm{MD}(\mathrm{dB})$ & $-0.11(-0.29,0.07)$ & 0.215 & $0.06(-0.29,0.33)$ & 0.744 & $0.16(-0.08,0.40)$ & 0.193 \\
\hline Refractive errors (SEQ) (per D) & $-0.03(-0.22,0.17)$ & 0.799 & $-1.27(-1.90,-0.64)$ & $<0.001$ & $-1.14(-1.77,-0.46)$ & $<0.001$ \\
\hline
\end{tabular}

Abbreviations: $M D$ mean deviation, $d B$ decibels, $S E Q$ spherical equivalent, $D$ diopter, $I T$ inferotemporal to superonasal direction, $S T$ superotemporal to inferonasal direction 
factors, including age, mean deviation, and refractive errors using multivariate analysis. Further investigation using a greater number of subjects, a longitudinal design, and other potential factors (e.g., central corneal thickness) will be required to confirm our findings. However, the effect of refractive errors was statistically significant and unlikely to be overestimated due to sample size. Second, we measured LC tilting within the optic disc cupping as the reflectivity of LC beneath the neuroretinal rim was weaker than that within the cupping. To exclude the possibility of incorrect measurements, we chose not to use the LC surface line beneath the rim. Visualization of the LC surface beneath the rim, if possible, would enable more accurate determination of LCTA. Third, we were unable to correct the magnification effects on lateral measurement of fundus structures through OCT imaging, which could affect the results of angle measurement. According to Littman [28], uncorrected lateral measurement is decreased with increasing axial length. Therefore, the possibility that magnification effects were, at least in part, responsible for the correlation between LCTA and refractive errors cannot be excluded. We estimated the effects of magnification error on angle measurement and measurement error to be less than $15 \%$ in the eyes of our current subjects. Thus, this effect would not have had a critical effect on our results as refractive errors were associated only with IT-LCTA using multivariate analysis, regardless of the identical magnification effects on IT-LCTA or ST-LCTA. However, the effects of axial length and refraction on morphological parameters should be investigated in further studies. In addition, the angles measured in our study using OCT should be viewed with caution, as they were calculated by imaging software and were not actual measured distances over the curvature of the eye. Nevertheless, our study is the first to report measurements obtained by OCT in eyes with myopia, and we believe that these measurements and associations will enhance our understanding of myopia and LC.

In conclusion, we obtained in-vivo $3 \mathrm{D}$ images of $\mathrm{BMO}$ and LC planes and measured inferior-temporal and superior-temporal tilt angles of LC plane from the BMO plane. Inferior-temporal LCTAs, and the difference between superior and inferior LCTAs, were found to be correlated with refractive errors. These results suggest that LC tilting against BMO was vertically asymmetrical, and the inferior temporal direction was more strongly correlated with refractive errors. These distortions may provide an additional explanation for the various disorders affecting the disc due to myopia; however, further studies are required to clarify the internal optic disc structural changes associated with this visual defect.
Acknowledgments The authors wish to thank Kobori for help in preparing the study and clinical input. This study was supported in part by the Takeda Science Foundation, Tokyo, Japan, and by the Japan Society for the Promotion of Science (JSPS) KAKENHI Grant Numbers 25870678, $15 \mathrm{~K} 04699$, and $15 \mathrm{~K} 21335$.

Contributions T.S., H.K., and S.Y. designed the study. T.S., M.S., and H.I. performed analysis. T.S. wrote the paper. T.S and H.I. prepared all figures. H.K and M.A reviewed the article. All authors contributed to the editing of the paper and to scientific discussions.

\section{Compliance with ethical standards}

Funding Takuhei Shoji provided financial support in the form of Takeda Science Foundation, Tokyo, Japan, and by the Japan Society for the Promotion of Science (JSPS) KAKENHI Grant Numbers 25870678 and $15 \mathrm{~K} 21335$, and Masayuki Suzuki provided financial support in the form of JSPA AKENHI Grant Numbers 15K04699.

Competing financial interests Takuhei Shoji (T.S.): Lecturer; Alcon Japan; Pfizer Japan; Senju; Santen; Kowa

Makoto Araie (M.A.): Advisor or Consultant; B \& M; Senju; Santen; Kowa; Pfizer Japan; Topcon; Alcon Japan; Allergan Japan; Heidelberg Engineering

Lecturer; Carl-Zeiss Meditec; Nitten; Otsuka; Heidelberg Engineering; Senju; Topcon; Aerie Patent; Topcon

Ethical approval All procedures performed in studies involving human participants were in accordance with the ethical standards of the institutional and/or national research committee and with the 1964 Helsinki Declaration and its later amendments or comparable ethical standards.

Informed consent Informed consent was obtained from all individual participants included in the study.

Open Access This article is distributed under the terms of the Creative Commons Attribution 4.0 International License (http:// creativecommons.org/licenses/by/4.0/), which permits unrestricted use, distribution, and reproduction in any medium, provided you give appropriate credit to the original author(s) and the source, provide a link to the Creative Commons license, and indicate if changes were made.

\section{References}

1. Marcus MW, de Vries MM, Junoy Montolio FG, Jansonius NM (2011) Myopia as a risk factor for open-angle glaucoma: a systematic review and meta-analysis. Ophthalmology 118:1989.e21994.e2. doi:10.1016/j.ophtha.2011.03.012

2. Chihara E, Sawada A (1990) Atypical nerve fiber layer defects in high myopes with high-tension glaucoma. Arch Ophthalmol 108: 228-232

3. Phelps CD (1982) Effect of myopia on prognosis in treated primary open-angle glaucoma. Am J Ophthalmol 93:622-628

4. Burgoyne CF, Downs JC, Bellezza AJ, Suh JK, Hart RT (2005) The optic nerve head as a biomechanical structure: a new paradigm for understanding the role of IOP-related stress and strain in the pathophysiology of glaucomatous optic nerve head damage. Prog Retin Eye Res 24:39-73. doi:10.1016/j.preteyeres.2004.06.001 
5. Quigley HA, Hohman RM, Addicks EM, Massof RW, Green WR (1983) Morphologic changes in the lamina cribrosa correlated with neural loss in open-angle glaucoma. Am J Ophthalmol 95:673-691

6. Reis AS, Sharpe GP, Yang H, Nicolela MT, Burgoyne CF, Chauhan BC (2012) Optic disc margin anatomy in patients with glaucoma and normal controls with spectral domain optical coherence tomography. Ophthalmology 119:738-747. doi:10.1016/j.ophtha.2011.09.054

7. Chauhan BC, Burgoyne CF (2013) From clinical examination of the optic disc to clinical assessment of the optic nerve head: a paradigm change. Am J Ophthalmol 156:218.e2-227.e2. doi:10.1016/j.ajo.2013.04.016

8. Park SC, Brumm J, Furlanetto RL, Netto C, Liu Y, Tello C, Liebmann JM, Ritch R (2015) Lamina cribrosa depth in different stages of glaucoma. Invest Ophthalmol Vis Sci 56:2059-2064. doi:10.1167/iovs.14-15540

9. Furlanetto RL, Park SC, Damle UJ, Sieminski SF, Kung Y, Siegal N, Liebmann JM, Ritch R (2013) Posterior displacement of the lamina cribrosa in glaucoma: in vivo interindividual and intereye comparisons. Invest Ophthalmol Vis Sci 54:4836-4842. doi:10.1167/iovs.12-11530

10. Kuroda H, Baba M, Suzuki M, Yoneya S (2013) Ultra-high sensitive and high resolution optical coherence tomography using a laser induced electromagnetic dipole. Appl Phys Lett 103:141118

11. Shoji T, Kuroda H, Suzuki M, Baba M, Araie M, Yoneya S (2015) Three-dimensional optic nerve head images using optical coherence tomography with a broad bandwidth, femtosecond, and modelocked laser. Graefes Arch Clin Exp Ophthalmol 253:313-321. doi:10.1007/s00417-014-2870-5

12. Shoji T, Kuroda H, Suzuki M, Baba M, Hangai M, Araie M, Yoneya S (2014) Correlation between lamina cribrosa tilt angles, myopia and glaucoma using OCT with a wide bandwidth femtosecond mode-locked laser. PLoS ONE 9: e116305. doi:10.1371/journal.pone.0116305

13. Anderson DR, Chauhan B, Johnson C, Katz J, Patella VM, Drance SM (2000) Criteria for progression of glaucoma in clinical management and in outcome studies. Am J Ophthalmol 130:827-829

14. Kuroda H, Baba M, Suzuki M, Yoneya S (2013) A high speed three-dimensional spectral domain optical coherence tomography with $<2 \mu \mathrm{m}$ axial resolution using wide bandwidth femtosecond mode-locked laser. Appl Phys Lett 102: 251102

15. Park HY, Jeon SH, Park CK (2012) Enhanced depth imaging detects lamina cribrosa thickness differences in normal tension glaucoma and primary open-angle glaucoma. Ophthalmology 119:10 20. doi:10.1016/j.ophtha.2011.07.033

16. Tan O, Chopra V, Lu AT, Schuman JS, Ishikawa H, Wollstein G, Varma R, Huang D (2009) Detection of macular ganglion cell loss in glaucoma by Fourier-domain optical coherence tomography. Ophthalmology 116(12): 2305-2314. doi:10.1016/j.ophtha.2009.05.025

17. Park SC, De Moraes CG, Teng CC, Tello C, Liebmann JM, Ritch R (2012) Enhanced depth imaging optical coherence tomography of deep optic nerve complex structures in glaucoma. Ophthalmology 119:3-9. doi:10.1016/j.ophtha.2011.07.012

18. Tay E, Seah SK, Chan SP, Lim AT, Chew SJ, Foster PJ, Aung T (2005) Optic disk ovality as an index of tilt and its relationship to myopia and perimetry. Am J Ophthalmol 139:247-252. doi:10.1016/j.ajo.2004.08.076

19. Kim TW, Kim M, Weinreb RN, Woo SJ, Park KH, Hwang JM (2012) Optic disc change with incipient myopia of childhood. Ophthalmology 119(1):21.e1-3-26.e1-3. doi:10.1016/j.ophtha.2011.07.051

20. Ohno-Matsui K, Akiba M, Moriyama M, Shimada N, Ishibashi T, Tokoro T, Spaide RF (2012) Acquired optic nerve and peripapillary pits in pathologic myopia. Ophthalmology 119:1685-1692. doi:10.1016/j.ophtha.2012.01.047

21. Takayama K, Hangai M, Kimura Y, Morooka S, Nukada M, Akagi T, Ikeda HO, Matsumoto A, Yoshimura N (2013) Threedimensional imaging of lamina cribrosa defects in glaucoma using swept-source optical coherence tomography. Invest Ophthalmol Vis Sci 54:4798-4807. doi:10.1167/iovs.13-11677

22. Toranzo J, Cohen SY, Erginay A, Gaudric A (2005) Peripapillary intrachoroidal cavitation in myopia. Am J Ophthalmol 140:731732. doi:10.1016/j.ajo.2005.03.063

23. Shimada N, Ohno-Matsui K, Yoshida T, Yasuzumi K, Kojima A, Kobayashi K, Futagami S, Tokoro T, Mochizuki M (2006) Characteristics of peripapillary detachment in pathologic myopia. Arch Ophthalmol 124:46-52. doi:10.1001/archopht.124.1.46

24. Kiumehr S, Park SC, Syril D, Teng CC, Tello C, Liebmann JM, Ritch R (2012) In vivo evaluation of focal lamina cribrosa defects in glaucoma. Arch Ophthalmol 130:552559. doi:10.1001/archopthalmol.2011.1309

25. Kimura Y, Akagi T, Hangai M, Takayama K, Hasegawa T, Suda K, Yoshikawa M, Yamada H, Nakanishi H, Unoki N, Ikeda HO, Yoshimura N (2014) Lamina cribrosa defects and optic disc morphology in primary open angle glaucoma with high myopia. PLoS ONE 9:e115313. doi:10.1371/journal.pone.0115313

26. Strouthidis NG, Yang H, Reynaud JF, Grimm JL, Gardiner SK, Fortune B, Burgoyne CF (2009) Comparison of clinical and spectral domain optical coherence tomography optic disc margin anatomy. Invest Ophthalmol Vis Sci 50:4709-4718. doi:10.1167/iovs.09-3586

27. Hosseini H, Nassiri N, Azarbod P, Giaconi J, Chou T, Caprioli J, Nouri-Mahdavi K (2013) Measurement of the optic disc vertical tilt angle with spectral-domain optical coherence tomography and influencing factors. Am J Ophthalmol 156:737-744. doi:10.1016/j.ajo.2013.05.036

28. Littmann $H$ (1982) Determination of the real size of an object on the fundus of the living eye. Klin Monatsbl Augenheilkd 180:286-289 